\title{
THEMATISCHER SCHWERPUNKT 2017: Entgrenzungen
}

Die Konjunktur von Fragen nach Funktionen und Bedeutung der Grenzen dauert fast seit einem halben Jahrhundert in vielen Forschungsdisziplinen an. Wichtige Impulse für die Entwicklung von border studies lieferten um die Jahrtausendwende 2000 demokratische Transformationen in Mitteleuropa, die nicht nur politisch-administrative Funktionen mittelosteuropäischer Grenzen nachhaltig veränderten, sondern auch zu einer intensiven Reflexion über deren historische, geographische, politische, soziologische, rechtliche und nicht zuletzt anthropologische Bedeutung anregten.

Die Prozesse in Mittelosteuropa können zu einem großen Teil als Prozesse der Entgrenzungen gesehen werden. Die Öffnung der Grenzen innerhalb des ehemaligen ,Ostblocks‘ und vor allem gegenüber dem ,Westen', der konsequente Abbau von Restriktionen, die sowohl die persönliche Mobilität als auch eine freie Gestaltung von Arbeits- und Handelsmärkten beeinträchtigten, begleitet von strategischen politischen Entscheidungen (Grenz- und Nachbarschaftsverträge, EU-Erweiterung etc.) haben sowohl praktisch als auch emotional die Bedeutung von Staatsgrenzen zu einer eher administrativ-theoretischen Notwendigkeit schrumpfen lassen. Nicht zuletzt dadurch trat die europäische sprachliche, kulturelle, historische und mentale Vielfalt stärker in Erscheinung, die aber nicht nur zu einer Reflexion über den verbindenden Charakter von Grenzen einlud, sondern auch Anlass zu einem neuen Grenzdenken bot. Die die europäische Entwicklung überlagernden Globalisierungsprozesse, die unter anderem eine Krise der Nationalstaaten oder ein wiedererwecktes Interesse an Imperien zur Folge haben (FRANÇOIS, SEIFARTH, STRUCK 2007:7), zeigen weitere Konsequenzen von Entgrenzungsprozessen auf - und zwar in einer großen Skala.

Das Auflösen und Demontieren von Grenzen scheint nicht nur in politischgeographischer Hinsicht ein Signum der Jahrtausendwende zu sein. Dabei sind Entgrenzungen nicht nur Gegenstand theoretischer Reflexionen, sondern auch ein Teil der kulturellen, sozialen oder sprachlichen Lebenspraxis. So werden Grenzen etwa identitätsstiftender Kollektive (Generationen, Nationen) weitgehend durchlässiger, auch Konsequenzen, die sich aus bisher mehr oder weniger als obligat angesehenen Dependenzverhältnissen ergaben, erscheinen immer häufiger als diskutabel (siehe etwa die postulierte Orthographiefreiheit auf Internetforen). Zu fragen wäre, inwiefern Entgrenzungsprozesse als Resultat des 
postmodernen Dekonstruktivismus anzusehen sind, der Grenzen von einem „harten und eindeutigen Schnitt“ (WOKART 1995:284) zu einem notwendigen Konstruktionselement von Begriffen und Kategorien umfunktionierte. Das seit etwa fünfzehn Jahren vielerorts diagnostizierte Ende der Postmoderne wird ein Prüfstein für die Nachhaltigkeit dieser Erkenntnis sein. Auch die Entwicklung von Informations- und Kommunikationstechnologien definieren die Grenzen neu: das Verschwinden von Peripherien im Internet, Möglichkeiten einer mehrfachen Präsenz des Subjekts, virtuelle Realität und Immersion werfen neue Fragen nach den Grenzen zwischen Realität und Simulation, Körper und Avatar, einem biologischen und einem digitalen Leben auf.

Die Entgrenzungsprozesse im oben erörterten Sinn wecken meist positive Emotionen. Werden Schranken aufgehoben, tun sich, so denkt man oft, auch neue Erscheinungsformen des Ästhetischen im künstlerischen Schaffen und somit auch neue Explorationsfelder für Literatur- und Kulturwissenschaftler auf. Die Vorstellung einer durch keinerlei dogmatische und formale Hindernisse beschränkten (Sprach-)Kunst hat eine nahezu magische Anziehungskraft für Ästheten und Schöngeister jeglicher Provenienz. Diese Faszination wird von Linguisten aber nicht geteilt: Wo die einen inhaltliche Freiheit und Freude am Ausdruck sehen, warnen die anderen vor Chaos und Anarchie beim Kommunizieren. Die Stabilität des Sprachsystems gilt für sie als tragender Pfeiler der sprachlichen Verständigung. Selbstverständlich werden die Grenzen des Sprachsystems oftmals angetastet, sei es im Akt einer kreativen Sprachverwendung, sei es durch sich verfestigende Fehler im Sprachgebrauch. Manche kreativen Ein- oder hartnäckigen Missgriffe gehen sogar im Laufe der Zeit in das System ein.

Unser thematischer Schwerpunkt lädt zu Stellungnahmen zu der Überlegung ein, inwieweit die Grenzen des Sprachstandards verschiebbar sind. Wo endet die Freiheit des Sprachbenutzers? Wo beginnt die Anarchie beim Kommunizieren? Was hat als unantastbar zu gelten? Wie viel Irreguläres und Ungrammatisches kann dem Kommunikationspartner zugemutet werden?

Convivium lädt zu einer kritischen Reflexion über Entgrenzungsprozesse innerhalb der Kultur und Sprache ein.

\section{Literatur}

FRANÇOIS, ETIENNE / SEIFARTH, JÖRG/ STRUCK, BERNHARD (2007): Einleitung: Grenzen und Grenzräume. Erfahrungen und Konstruktionen. In François, ETIENNE / SEIFARTH, JÖRG/ StRUCK, BERNHARD (eds.): Die Grenze als Raum, Erfahrung und Konstruktion. Frankfurt/New York: 7-29. 
WOKART, NORBERT (1995): Differenzierungen im Begriff „Grenze“. In: FABER, RICHARD / NAUMANN, BARBARA (eds.): Literatur der Grenze - Theorie der Grenze. Würzburg: 275-298. 